\title{
Wavelength Dependence of the Polarization Singularities in a Two-Mode Optical Fiber
}

\author{
V. V. G. Krishna Inavalli, Vijay Pottapinjara, and Nirmal K. Viswanathan \\ School of Physics, University of Hyderabad, Hyderabad 500 046, India \\ Correspondence should be addressed to Nirmal K. Viswanathan, nirmalkv@hotmail.com
}

Received 14 May 2011; Revised 3 July 2011; Accepted 7 July 2011

Academic Editor: Shunichi Sato

Copyright ( $) 2012$ V. V. G. Krishna Inavalli et al. This is an open access article distributed under the Creative Commons Attribution License, which permits unrestricted use, distribution, and reproduction in any medium, provided the original work is properly cited.

\begin{abstract}
We present here an experimental demonstration of the wavelength dependence of the polarization singularities due to linear combination of the vector modes excited directly in a two-mode optical fiber. The coherent superposition of the vector modes excited by linearly polarized Gaussian beam as offset skew rays propagated in a helical path inside the fiber results in the generation of phase singular beams with edge dislocation in the fiber output. The polarization character of these beams is found to change dramatically with wavelength-from left-handed elliptically polarized edge dislocation to right-handed elliptically polarized edgedislocation through disclinations. The measured behaviour is understood as being due to intermodal dispersion of the polarization corrections to the propagating vector modes, as the wavelength of the input beam is scanned.
\end{abstract}

\section{Introduction}

Singular optics is the new branch of modern physical optics that deals with a wide class of effects associated with phase singularities in wave fields and topology of wave fronts [1]. There are three levels of optical singularities: ray singularities (caustics), singularities of plane polarized waves (scalar fields), and polarization singularities of vector light fields that are extensively researched in the recent times as several new possibilities for applications are being explored $[2,3]$. Among the most interesting objects of research in singular optics are the polarization singularities (PS), arising in spatially inhomogeneously polarized light fields $[2,3]$. The singular structure of such a field is formed by the disclination of the wavefront ( $d$-singularities), as well as by $C$-points and $L$-contours observed in the plane perpendicular to the direction of propagation of a paraxial optical beam [4]. PSs are studied extensively in fully developed speckle (random) fields, either from a highly scattering medium or in the output from a multimode optical fiber $[3,5]$. Though the random fields are a research area of fundamental importance to study the generic features of optical singularities, their properties are highly statistical in nature and at this point appear to have limited applications [2, 3].
First realized by Zel' dovich et al., the output radiation field from a multimode optical fiber is replete with forklike interference structure, indicative of its vortical nature [6]. In 2002 Volyar demonstrated that the appearance of optical vortices in the low-mode fiber output are due to the existence of guided modes and discussed their main features [5]. Based on their extensive work on fiber singular optics and subsequently extended by Alexeyev et al., to study the propagation of optical vortices in weakly guiding perturbed fibers [7] it is now well established that the radiation field of low-mode optical fibers exhibit singularity points, whose position depends on the excitation conditions $[8,9]$, external perturbations, and orientation of polarizer at the output of the fiber [10]. The number of guided modes excited in the fiber and their weights are important to understand and analyze the formation of singularity in the fiber output. One of the methods is where the mode composition is analyzed qualitatively by comparing the output radiation pattern with numerically simulated intensity distributions [11]. In the case of two-mode fibers the mode groups with same azimuthal $(l)$ and radial $(m)$ indices are excited and the mode weights from within the group of same index "l" with a singularity in the radiation field are considered [5]. It is also 
important to remember that the intensity distributions in the fiber output are affected by the mode dispersion arising from within the mode group [12].

In addition, in optical fibers, as a rule, the input polarization changes by virtue of the birefringence of the medium [13]. For a fixed linear input polarization the evolution of polarization along the fiber with residual linear birefringence is measured either by cutback technique or wavelength scanning method $[14,15]$. The measured wavelength dependence of the output polarization state moves along a trajectory on the Poincare sphere $[16,17]$. In addition, for skew rays launched into the fiber, the polarization vector of the light ray rotates as it propagates along a twisted path in the inhomogeneous fiber medium [18]. Skew rays are launched into the fiber by adjusting the position and the angle of illumination at the fiber input end to predominantly excite rays with right or left helicity $\exp ( \pm i l \phi)$ modes of the axially symmetric fiber [19]. The birefringence properties of the step-index optical fiber are observed as a difference in the polarization corrections $\delta \beta$ to the propagation constants $\tilde{\beta}$ of the azimuthally symmetric linearly polarized OV (LV) or circularly polarized (CP) mode [20]. The intermodal dispersion due to the difference in the propagation constants of the fiber modes and their polarization correction leads to vector inhomogeneity of the output field from the fiber $[7,20]$.

In this paper we present our experimental results on the wavelength dependence of the vector inhomogeneity and hence of the polarization singularity generated in the output of a two-mode optical fiber (TMF). The input excitation conditions are so chosen to result in a combination of right and left elliptically polarized two-lobe output intensity pattern via selective excitation of the guided vector-vortex modes of opposite charges. The elliptically polarized singular beam is formed due to coherent coaxial mixing of weighted orthogonally polarized single-charged Laguerre-Gauss (LG) modes of the fiber. As the wavelength of the input beam is scanned over a range from 740-760 nm we track the changes in the intensity, phase, and polarization of the output beam from the TMF. The phase and polarization character of the output beam from the TMF are characterized using the interferometer and Stokes parameter measurements. A custom Matlab code is developed to map the polarization ellipses, calculated from the measured Stokes parameters, directly onto the output beam to follow the polarization evolution of different parts of the beam as the input beam wavelength is scanned. Using the normalized Stokes parameters $\left(S_{1} / s_{0}, S_{2} / s_{0}\right.$, and $\left.S_{3} / s_{0}\right)$ the trajectory at a point of the elliptically polarized output beam from the TMF is tracked on the Poincare sphere as a function of input wavelength. The output beam intensity and the polarization ellipse parameters $(b, \psi)$ show periodic behaviour, clearly indicating change in the rotation direction of the polarization ellipse from right to left through linear polarization as a function of wavelength of the input beam, in the linear regime of operation. Thus, the polarization character of the output beam from the TMF is found to change dramatically-from left-handed elliptically polarized edge-dislocation into a right-handed elliptically polarized-edge dislocation through disclinations. The measured behaviour is understood to be as due to intermodal dispersion of the vector modes and their polarization correction as the wavelength of the input beam is scanned. The wavelength scanning method for such measurements has the added advantage that the orientation of the fiber is not altered during the measurements and is a nondestructive method.

\section{Experimental Details}

Schematic of the experimental setup used for the generation of polarization singular beams using a TMF is shown in Figure 1 along with the setup to characterize the phase and polarization singularities of the beam. We first generate the desired singular beam at a given wavelength, carry out the required interference and Stokes parameters measurements on the beam, and repeat the same for different wavelengths spaced uniformly. A tuneable continuous-wave (CW) Ti: Sapphire laser (MBR 110, Coherent, USA) is used as the source of light. The overall wavelength range of operation of the laser is $700 \mathrm{~nm}-1000 \mathrm{~nm}$ and the range of interest to us here is $740-760 \mathrm{~nm}$, which is in the middle of the twomode regime $(2.4048<V<3.8317)$ of the fiber. The laser output is linearly polarized (500:1) TEM $_{00}$ mode. A 4\% reflection from the glass plate is taken and coupled to an Optical Spectrum Analyzer (AQ 6370B, Yokogawa, Japan) to monitor the wavelength of the light. A Glan-Thomson polarizer (GTP) $\left(>10^{5}: 1\right)$ and a half-wave plate (HWP) are used to have a better control on the polarization of the input beam launched into the TMF (HI 980 fiber of length $32.2 \mathrm{~cm}$ ) thereby controlling the output beam characteristics. A modified Mach-Zender interferometer is constructed in parallel to measure the phase characteristics of the generated beam. An $R / T=50 / 50$ beam splitter is used to split the incoming light beam into two beams of almost equal intensity. The beam passing through the beam-splitter $\left(\mathrm{BS}_{1}\right)$ is focused using a microscope objective lens $\mathrm{L}_{1}(0.20 \mathrm{NA}$ and $10 \mathrm{x})$ to couple the light into the fiber, whose input end is fixed on a 3-axis precision launching mount. The output end of the fiber is held using a v-groove clamp and the light output from the fiber is collimated using another objective lens $\mathrm{L}_{2}(0.40 \mathrm{NA}$ and $20 \mathrm{x})$ as shown in Figure 1 . The reference beam that is reflected off the $\mathrm{BS}_{1}$ and the output beam from the fiber are made almost collinear using the beam splitter $\left(\mathrm{BS}_{2}\right)$. A CCD camera connected to computer via IEEE 1394 card is used to capture the images of the light beam at the fiber and the interferometer output. A stop (S) is used to block the reference beam whenever it is not required.

The desired output beam (with embedded dislocations and disclinations) from the TMF is produced by adjusting the controls-HWP orientation angle, and the launching conditions at a given wavelength of the input beam and for a fixed fiber length, as discussed in [9]. The images of the direct output beam from the TMF and the corresponding interference pattern are first captured using the CCD camera. The Stokes parameter of the beam is calculated using the six images captured by first introducing a polarizer (with its axis 


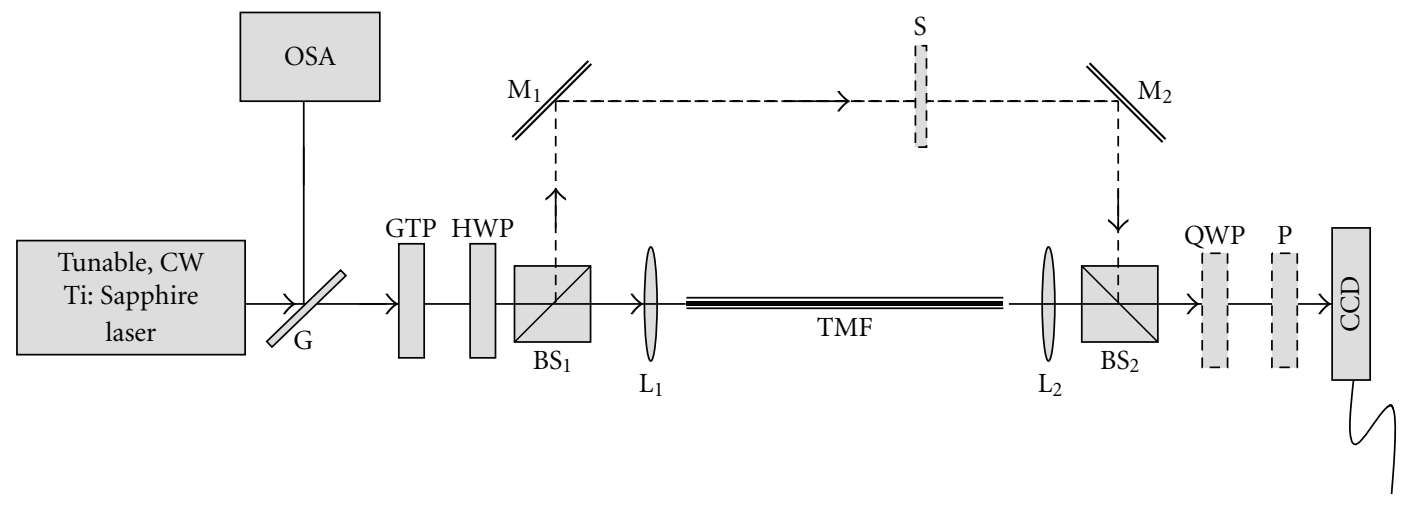

FIGURE 1: Schematic of the experimental setup use to generate and manipulate polarization singular beams using a two-mode optical fiber. G: Glass Plate; GTP: Glan-Thomson Polarizer; HWP: Half-Wave Plate; BS: Beam-splitter; L: Objective Lens; S: Stop; QWP: Quarter-Wave Plate; P: Polarizer; CCD: Camera.

oriented at $0^{\circ}, 45^{\circ}, 90^{\circ}$, and $135^{\circ}$ ) and then with the QWP (at $45^{\circ}$ and $135^{\circ}$ ) and polarizer (oriented at $90^{\circ}$ ) combination. Without disturbing the launching conditions or the orientation of the polarization sensitive optical components in the setup, the wavelength of the laser is scanned from 740$760 \mathrm{~nm}$ in steps of $1 \mathrm{~nm}$ (by tuning the birefringent filter in the laser cavity) and the above-mentioned measurements are repeated for all the output beams. From the measurements we observe that the modal characteristics (both intensity and polarization) of the beam changes dramatically with the input laser wavelength as the propagation constants $\left(\beta_{i}\right)$ of the vector modes are wavelength dependent.

\section{Results and Discussion}

For the input wavelength of the Ti: Sapphire laser tuned to $\lambda_{1}$ $=740 \mathrm{~nm}$ and the HWP oriented at $0^{\circ}$, the linear vertically polarized Gaussian beam is coupled into the cleaved input end of the TMF. By placing the fiber at the focal point of the objective lens $L_{1}$ and adjusting the " $x$ " and " $y$ " positions of the fiber we launch offset skew rays into the fiber. The output two-lobe intensity pattern is similar to the $\mathrm{HG}_{01}$ mode of laser resonators with a $\pi$-phase shifted interference pattern between the lobes measured using the CCD camera and shown in Figures 2(a) and 2(b). Assuming that the linear vertically polarized Gaussian input beam coupled into the TMF excites the CP mode $|\mathrm{CP}(0)\rangle=\left(\begin{array}{l}1 \\ i\end{array}\right) \cos \varphi$ within the first few millimetre of the fiber, the mode at the output of the fiber of length "l" can be written as being due to the superposition of the fiber's modes as [20]

$$
|\mathrm{CP}(z=l)\rangle=\frac{1}{2}\left[|1,1,1\rangle e^{i \beta_{1} l}+\left|\mathrm{TM}_{01}\right\rangle e^{i \beta_{4} l}+i\left|\mathrm{TE}_{01}\right\rangle e^{i \beta_{3} l}\right] .
$$

Assuming that there is no spin-orbit interaction between the excited modes the distribution of the field corresponding to the field looks like $I_{\mathrm{CP}}(z) \propto 2 \cos ^{2}(\varphi-\alpha l)$ and rotates by an angle " $\alpha l$ " during the propagation through the fiber or when the wavelength is changed due to the dispersion of the propagation constants of the modes excited [20]. However, the propagation of the excited fiber modes along a twisted path also changes the polarization distribution of the ideal linearly polarized modes due to the fiber birefringence.

Next, from the six polarization measurements on the output beam we calculate the Stokes parameters as shown in Figure 2(c). The scale of each Stokes parameter map (Figure 2(c)) is custom set to highlight that even small differences in the data will not be lost. As can be seen from the $\mathrm{S}_{3}$ Stokes parameter map, there is a small part of the left lobe which is right circularly polarized and the same is true for the other lobe and these portions are on diagonally opposite parts of the mode. The orientation of the ellipses is read form the $S_{1}$ and $S_{2}$ Stokes parameters. Overall, the part right- and left-handed elliptically polarized (REP/LEP) beam measured at the fiber output shown in Figure 1(d) is the resultant of the superposition between the vector modes excited in the fiber by linearly polarized rotating beam inside the fiber, akin to the propagation of linearly polarized optical vortex $|\mathrm{LV}(z)\rangle$ in the TMF given by [20]

$$
|\mathrm{LV}(z=l)\rangle=\frac{1}{2}\left[|1,1,1\rangle e^{i \beta_{1} l}+\left|\mathrm{TM}_{01}\right\rangle e^{i \beta_{4} l}-i\left|\mathrm{TE}_{01}\right\rangle e^{i \beta_{3} l}\right] .
$$

The resulting polarization distribution in the output beam is complicated due to the presence of spin-orbit interaction, especially as our fiber length $l \geq z_{0}$ [20].

From the six polarization measurements we calculate the Stokes parameters $\left(S_{0}-S_{3}\right)$ (Figure 2(c)) using which we construct the pointwise state of polarization (SOP) of the entire beam. The polarization ellipse parameters are given by $a=1, b= \pm \tan \chi, \psi=0.5 \tan ^{-1}\left(S_{2} / S_{1}\right)$, where " $a$ ", " $b$ ", and " $\psi$ " are the lengths of semimajor and semiminor axes and the orientation angle of the ellipse with respect to major axes, respectively [21]. From the intensity values at each pixel in the images captured by the CCD camera the Matlab code written for the purpose of calculating the polarization ellipse reads the image as a matrix with intensity values as its elements. The Stokes parameters and polarization ellipse parameters calculations are performed using these intensity values in the data images. The ellipse parameters are calculated at each point in the resized (based on the source correlation parameters) image, 


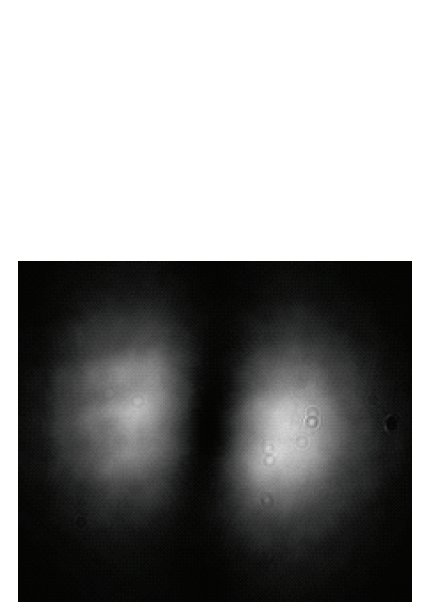

(a)

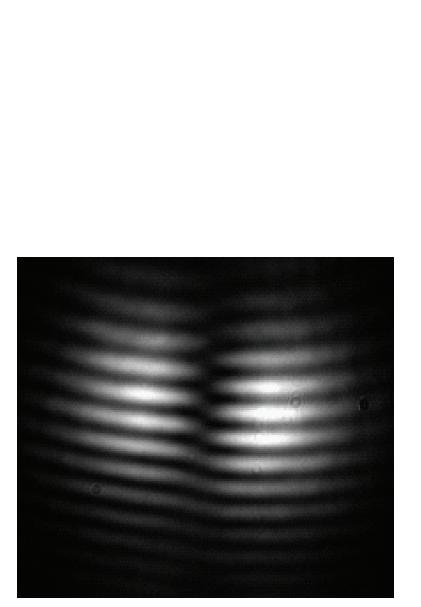

(b)

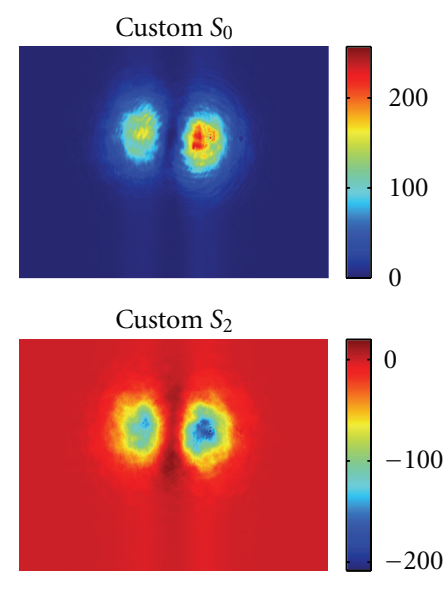

(c)

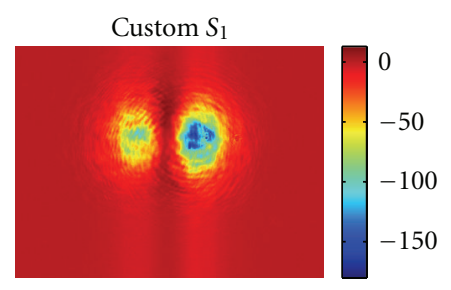

Custom $S_{3}$

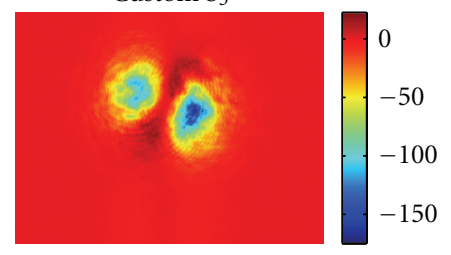

)

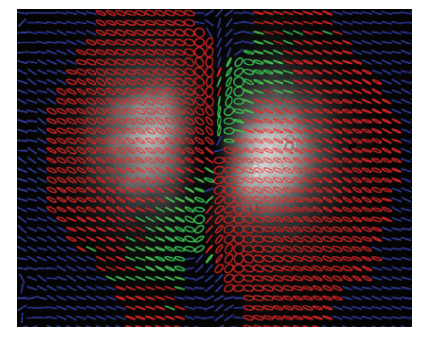

(d)

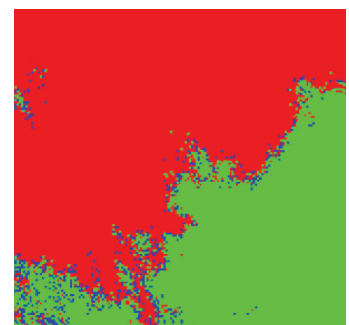

(e)

FIGURE 2: Intensity, phase, and polarization characteristics of the output beam from the TMF. (a) Two-lobe intensity profile, (b) $\pi$-phase shifted interference pattern of the output beam with unpolarized reference beam, (c) calculated Stokes parameters of the output beam from the six related measurements, (d) calculated polarization ellipse overlapped on the beam intensity pattern: green-right elliptical polarized, red-left elliptical polarized, and blue-linear polarization or no light, (e) high-resolution polarization ellipse map of a small portion of the pattern in (d).

and polarization ellipse is plotted overlaying the image that is of the same size as the original images. The point-wise polarization ellipses are mapped onto the direct beam images at fiber output for all input wavelengths of the laser. Also, as plotting the polarization ellipse at each pixel will make the polarization map blurred, we average the data over a block of $16 \times 16$ pixels (dimensions less than the source correlation length) and then the ellipse parameters are calculated at each point in the resized image. The fiber output intensity overlapped with the polarization ellipse calculated from the Stokes parameters is shown in Figure 2(d) to be lefthanded elliptically polarized mode mixed with right-handed elliptically polarized mode. The high-resolution calculation of the SOP of the beam at the interface between the two opposite elliptically polarization state shows linear polarized boundary (Figure 2(e)), as expected.

The output beam from the TMF is elliptically polarized due to the intramodal phase difference accumulated by the excited and propagated modes due to the fiber birefringence. In addition, the orientation of the polarization ellipse depends on the weight factor of the modes superposed to generate the output beam. These are governed by the overall residual linear birefringence of the TMF which includes inhomogeneities in the core shape, doping inhomogeneities which in turn give rise to nonuniform refractive index profile along the propagation direction, and so forth. The phase change introduced by the birefringence is given by $\Phi=$ $(2 \pi l / \lambda) \Delta n$, where $\Delta n$ is the birefringence (assumed wavelength independent, for closely spaced input wavelengths), $\lambda$ is the input wavelength, and $l$ is the fiber length. In the wavelength scanning method used here the wavelength dependence of the birefringence is neglected [16].

Next, without changing the input launch conditions we tune the laser wavelength in steps of $1 \mathrm{~nm}$ up to $\lambda_{f}=760 \mathrm{~nm}$. As the wavelength of the input laser is increased, part of the beam that is right elliptically polarized gradually increases in size, that is, the handedness change is happening from left to right in some parts of the output beam. From $\lambda=740 \mathrm{~nm}$ to $758 \mathrm{~nm}$ the above-mentioned change from LEP to REP over the entire beam is complete and at $\lambda=758 \mathrm{~nm}$ the entire beam is right elliptically polarized (Figure $3(\mathrm{a})$ ). Now moving beyond, at $\lambda=760 \mathrm{~nm}$ we see parts of the beam that are REP begin to turn around to being LEP. This confirms that the change in the polarization content (or change in the handedness) of the output beam is periodic. The boundary between the left-handed ellipse and right-handed ellipse, the $L_{x}$ and $L_{y}$ disclinations are measured by making the Stokes parameter $S_{3}=0$ (without the pixel average). For all input wavelengths we measure the SOP and the disclinations $L_{x}$ and $L_{y}$ of the output modes. The formation of elliptically 


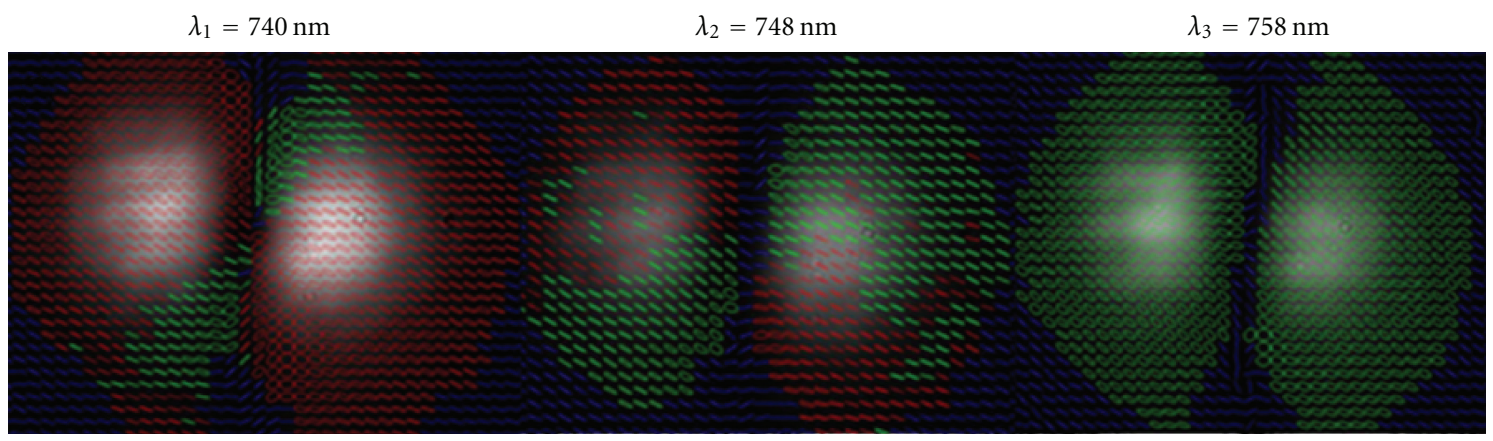

(a)
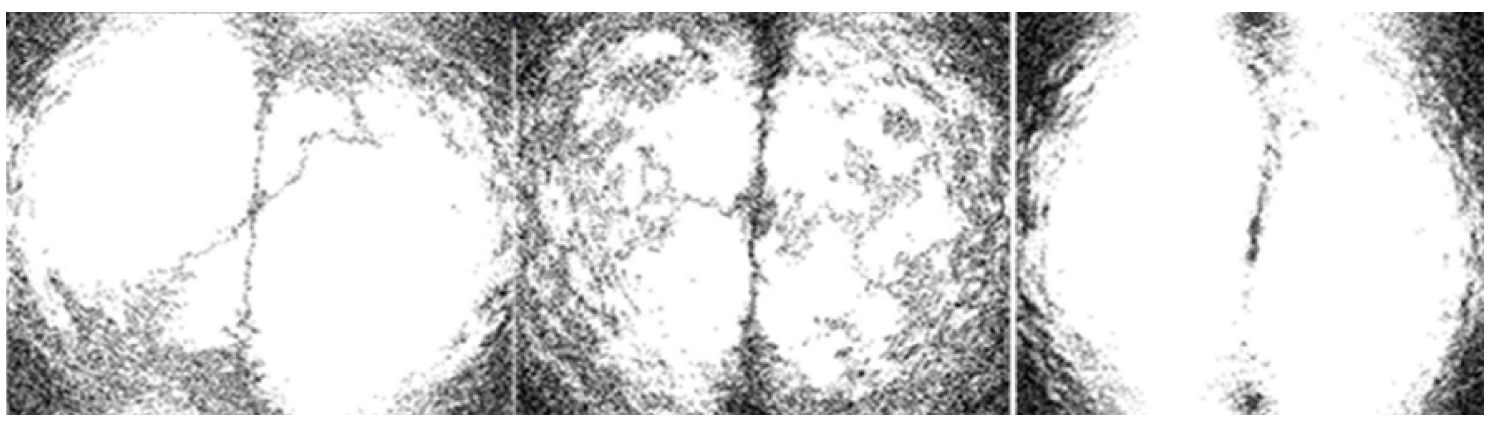

(b)

FIGURE 3: (a) Evolution of the polarization ellipse of the output beam from the TMF as a function of the input wavelength, (b) polarization line singularity (L-line) map as a function of the wavelength, indicating the evolution of dislocation via disclinations in the output beam from the TMF.

polarized edge dislocation due to the cyclic changes in the fiber modes are as shown in Figure 3(a) for three different input wavelengths of 740,748 , and $758 \mathrm{~nm}$, respectively. The evolutions of linearly polarized disclinations are shown as dark lines in Figure 3(b). It is interesting to note that when the input wavelength is tuned to $758 \mathrm{~nm}$, the linearly polarized disclinations are oriented in the same axis to form pure edge dislocation as shown in Figure 3.

In addition to plotting the polarization ellipse in the beam cross section we also plot the change in the output beam intensity along a fixed line across the beam for all the wavelengths (Figure 4). It is important to note here that the line profile of the two-lobe beam output from the fiber at the starting wavelength $\left(\lambda_{i}\right)$ has a slight asymmetry in its peak intensity, possibly due to the simultaneous presence of the fundamental fiber mode, $\mathrm{HE}_{11}$. However, a closer look at the polarization ellipse map (Figure $2(\mathrm{~d})$ ) does not indicate the presence of vectorial topological dipole and the output beam is free of any coarse speckle structure, a strong indication that there is no measurable residual fundamental $\mathrm{HE}_{11}$ mode leaking into the fiber output [22]. We believe that an almost complete filtering of the $\mathrm{HE}_{11}$ fiber mode was achieved in our experiment via a combination of off-axis launch [12], fiber v-clamp in the output, and a slight bend in the fiber along its length, which causes a slight asymmetry in the output two-lobe intensity pattern. A plot of the line profile of the output beam as a function of wavelength emphasizes that the beam intensity variation is periodic with wavelength (inset in Figure 4), possibly due to the dispersion of the polarization

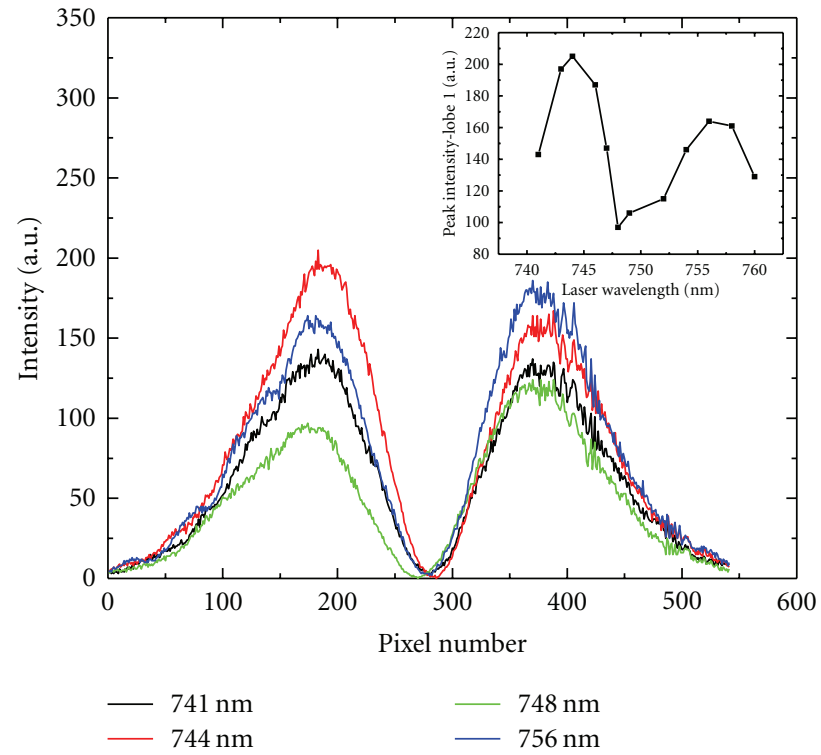

Figure 4: Line intensity plot of the output beam from the TMF as a function of the input wavelength. Inset shows the oscillating behaviour of the peak intensity of the left lobe as a function of the laser wavelength.

correction of the vector modes contributing to the output beam from the weakly birefringent elliptic TMF. Also, as the fiber vector modes are excited by offset and skew (linearly 


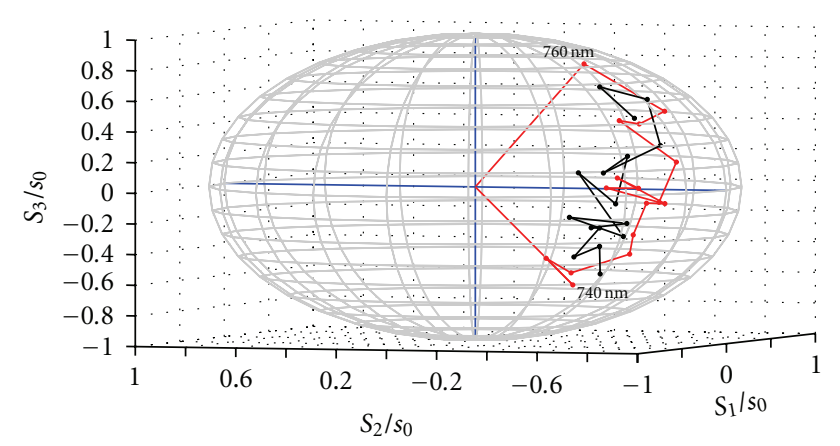

FIgURE 5: Poincare representation of the Stokes parameters measured in the TMF output as a function of the input laser wavelength. The two sets of data are for the left and right lobes of the output beam.

polarized) Gaussian beam that travels along a twisted path inside the fiber, the role of transverse energy flow in the field resulting in the rotation of the instantaneous energy distribution with wavelength cannot be ruled out [23]. Also, the small modulation contrast ( $\sim 100$ counts $)$ in the twolobe intensity pattern due to wavelength scanning further emphasizes that the dispersion in the polarization correction terms is of consequence here rather than the $\mathrm{HE}_{11}$ mode influence [24]. Thus it is clear from our observations that both the handedness of the polarization ellipse and intensity of the output beam changes periodically as the wavelength of the input beam is increased.

It is known that graphic representation of the polarization data permit a clear insight into a complex situation where the SOP evolution is tracked as a function of the wavelength. Based on the Stokes parameters calculated for the different input wavelengths we extract necessary information to plot the evolution in the Poincare sphere representation for both lobes of the output beam (Figure 5) [17]. For the fixed input linear polarization, scanning the input wavelength from $740 \mathrm{~nm}$ to $760 \mathrm{~nm}$ at a fixed point on the output beam, the SOP moves from REP to LEP, that is, from the bottom half of the sphere to the top half through the equator, corresponding to linear polarization state. As our wavelength scan range covers only a phase change of $\pi$ between the superposed vector modes, we have plotted only one half of the curve. However, proceeding with the increasing wavelength, we expect to cover the second half of the trajectory as well, which should correspond to the evolution of elliptically polarized light on a Poincaré sphere [25]. A plot of the polarization ellipse parameters ( $b$ and $\psi)$ extracted from the above measurements and calculations also shows periodic behaviour with wavelength as shown in Figure 6.

\section{Conclusion}

We presented here, to the best of our knowledge, a first experimental demonstration of the wavelength dependence of optical beams with polarization singularity generated using a two-mode fiber. The evolution of left elliptically polarized edge dislocation to pure right elliptically polarized

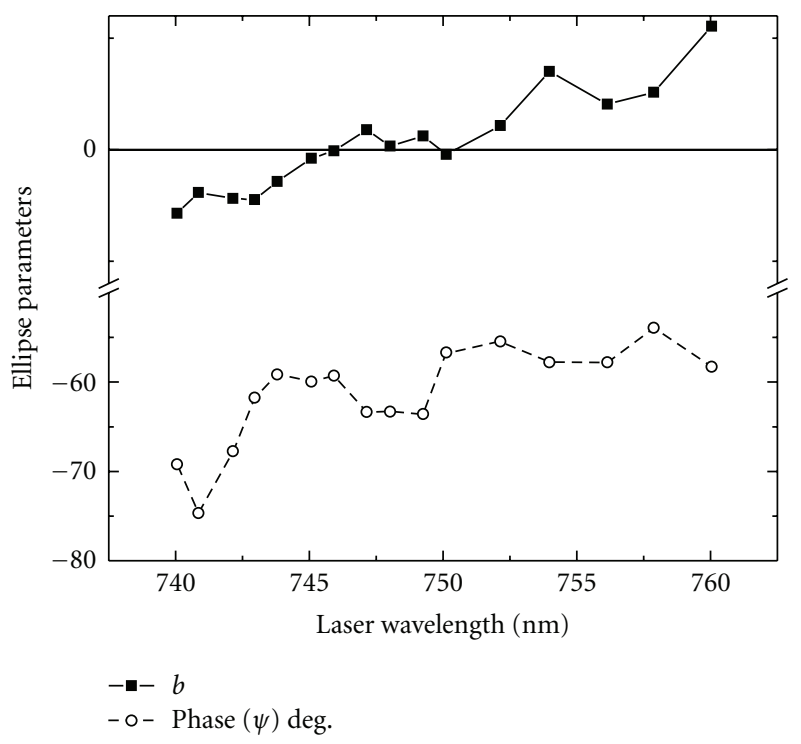

FIGURE 6: Ellipse parameters of the output beam from the TMF showing periodic behaviour as a function of input laser wavelength.

edge dislocation through disclinations is achieved by tuning the input laser wavelength in steps of $1 \mathrm{~nm}$. The changes in the polarization characteristics of these beams with the change in the wavelength occur through the dispersion of the propagation constant $\left(\beta_{i}\right)$ and the polarization correction $\left(\delta \beta_{i}\right)$ of the vector modes excited and propagated in the fiber. Some evidence is also shown to emphasize that these changes in addition to the intensity variations of the twolobe intensity pattern and the polarization ellipse parameters are cyclic with wavelength of the input beam. We anticipate that the work will be useful in the advancement of the applicability of the polarization singular beams, especially in polarization tweezers.

\section{Acknowledgments}

The authors acknowledge Department of Science and Technology (DST), Government of India for financial support for the project. V. V. G. K. Inavalli acknowledges CAS-RFSMS, UoH for research fellowship.

\section{References}

[1] M. S. Soskin and M. V. Vasnetsov, "Singular optics," in Progress in Optics, E. Wolf, Ed., vol. 42, chapter 4, pp. 219-276, Elsevier, Amsterdam, The Netherlands, 2001.

[2] M. R. Dennis, K. O. Holleran, and M. J. Padgett, "Singular optics: optical vortices and polarization singularities," in Progress in Optics, E. Wolf, Ed., vol. 53, chapter 5, pp. 293-359, Elsevier, Amsterdam, The Netherlands, 2009.

[3] O. V. Angelsky, Optical Correlation: Techniques and Applications, SPIE Press, Washington, Wash, USA, 2007.

[4] J. Nye, Natural Focusing and the Fine Structure of Light: Caustics and Wave Dislocations, IOP, Bristol, UK, 1999.

[5] A. V. Volyar, "Fiber singular optics," Ukrainian Journal of Physical Optics, vol. 3, no. 2, pp. 69-96, 2002. 
[6] B. Ya Zel'dovich, N. F. Pilipetsky, and V. V. Shkunov, Principles of Phase Conjugation, Springer Series in Optical Sciences, chapter 3, Springer, New York, NY, USA, 1985.

[7] C. N. Alexeyev, A. V. Volyar, and M. A. Yavorsky, "Fiber optical vortices," in Lasers, Optics and Electro-Optics Research, L. I. Chen, Ed., Nova Science Publishers, 2007.

[8] A. N. Alekseev, A. V. Volyar, and T. A. Fadeeva, "Eigenmode geometry in a high-birefringence optical fiber," Technical Physics Letters, vol. 31, no. 5, pp. 374-377, 2005.

[9] N. K. Viswanathan and V. V. G. K. Inavalli, "Generation of optical vector beams using a two-mode fiber," Optics Letters, vol. 34, no. 8, pp. 1189-1191, 2009.

[10] T. A. Fadeyeva and A. V. Volyar, "Tunneling selection of optical vortices,” Technical Physics Letters, vol. 29, pp. 594-597, 2003.

[11] C. Kerbage, P. S. Westbrook, R. S. Windeler, and B. J. Eggleton, "Modal characteristics of an air-silica microstructure fiber," Optics and Photonics News, vol. 12, no. 12, p. 18, 2001.

[12] A. W. Snyder and J. D. Love, Optical Waveguide Theory, Chapman and Hall, London, UK, 1983.

[13] I. Kaminow, "Polarization in optical fibers," IEEE Journal of Quantum Electronics, vol. 17, no. 1, pp. 15-22, 1981.

[14] K. Kikuchi and T. Okoshi, "Wavelength-sweeping technique for measuring the beat length of linearly birefringent optical fibers," Optics Letters, vol. 8, pp. 122-123, 1983.

[15] T. I. Su and L. Wang, "A cutback method for measuring low linear fibre birefringence using an electro-optic modulator," Optical and Quantum Electronics, vol. 28, no. 10, pp. 13951405, 1996.

[16] W. Eickhoff, Y. Yen, and R. Ulrich, "Wavelength dependence of birefringence in single-mode fiber," Applied Optics, vol. 20, no. 19 , pp. 3428-3435, 1981.

[17] F. T. Martinez, D. Tentori, C. A. Diaz, and F. J. M. Jimenez, "Birefringence assessment of single-mode optical fibers," Optics Express, vol. 13, no. 7, pp. 2556-2563, 2005.

[18] A. Y. Savchencko and B. Y. Zel'dovich, "Wave propagation in a guiding structure: one step beyond the paraxial approximation," Journal of the Optical Society of America B, vol. 13, no. 2, pp. 273-281, 1996.

[19] M. A. Bolshtyansky, A. Y. Savchenko, and B. Y. Zel'dovich, "Use of skew rays in multimode fibers to generate speckle field with nonzero vorticity," Optics Letters, vol. 24, no. 7, pp. 433435, 1999.

[20] C. N. Alexeyev, A. V. Volyar, and M. A. Yavorsky, "Optical vortices in twisted optical fibres with torsional stress," Journal of Optics A, vol. 10, no. 9, Article ID 095007, 2008.

[21] M. Born and E. Wolf, Principles of Optics, Cambridge University Press, Cambridge, UK, 6th edition, 1999.

[22] T. A. Fadeeva and A. V. Volyar, "Vectorial topological dipole in output radiation of a fiber optical coupler," Technical Physics Letters, vol. 30, no. 7, pp. 553-556, 2004.

[23] C. N. Alexeyev, E. V. Borshak, A. V. Volyar, and M. A. Yavorsky, "Angular momentum conservation and coupled vortex modes in twisted optical fibres with torsional stress," Journal of Optics A, vol. 11, no. 9, Article ID 094011, 2009.

[24] A. S. Jazi and J. C. McKeeman, "Synthesis of intensity patterns in few-mode optical fibers," Journal of Lightwave Technology, vol. 9, no. 9, pp. 1047-1052, 1991.

[25] F. Treviño-Martínez, D. Tentori, C. Ayala-Díaz, and F. J. Mendieta-Jiménez, "Birefringence assessment of single-mode optical fibers," Optics Express, vol. 13, no. 7, pp. 2556-2563, 2005. 

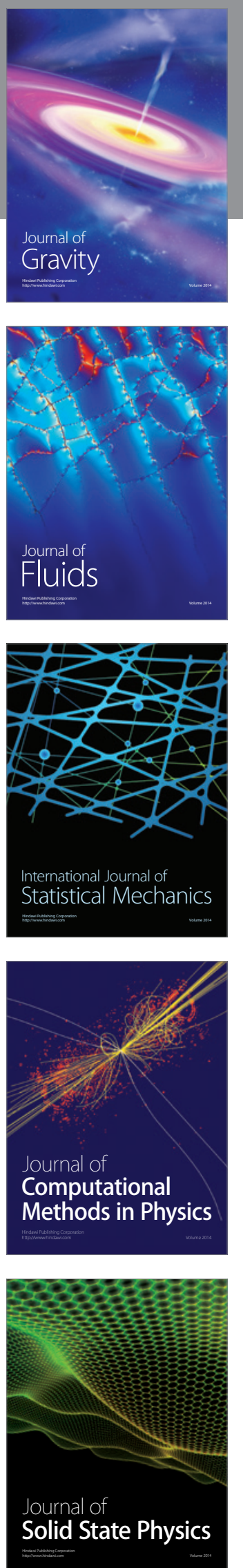

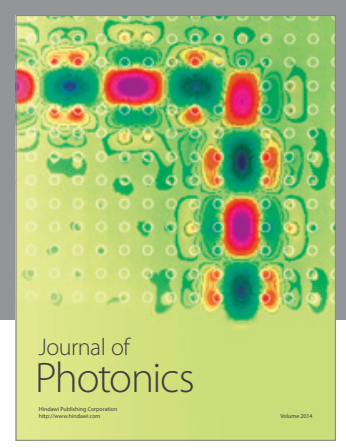

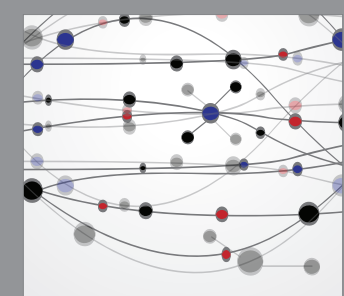

The Scientific World Journal
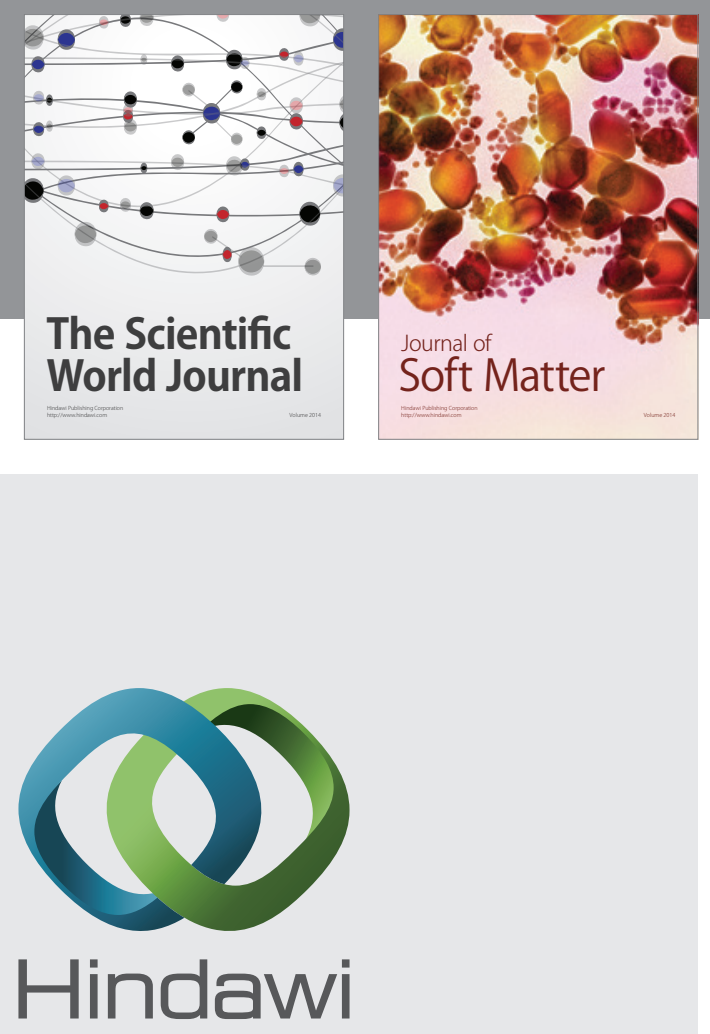

Submit your manuscripts at

http://www.hindawi.com
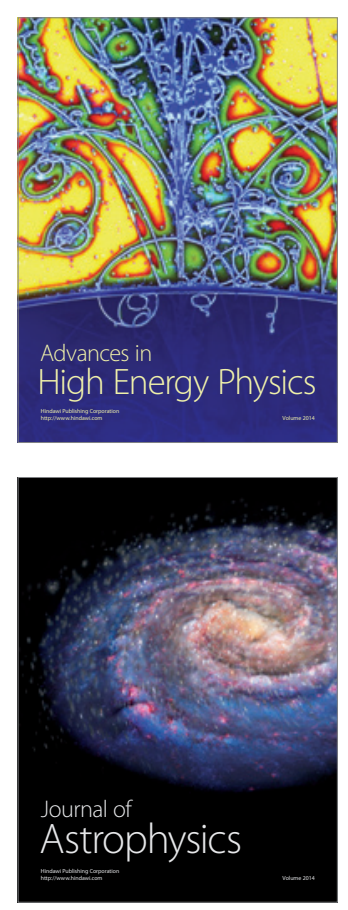
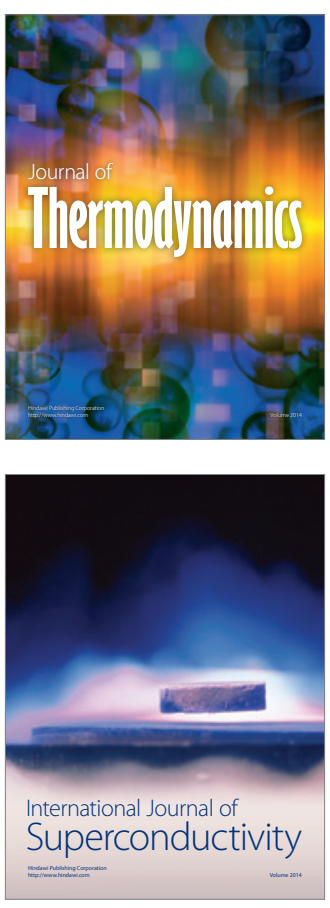
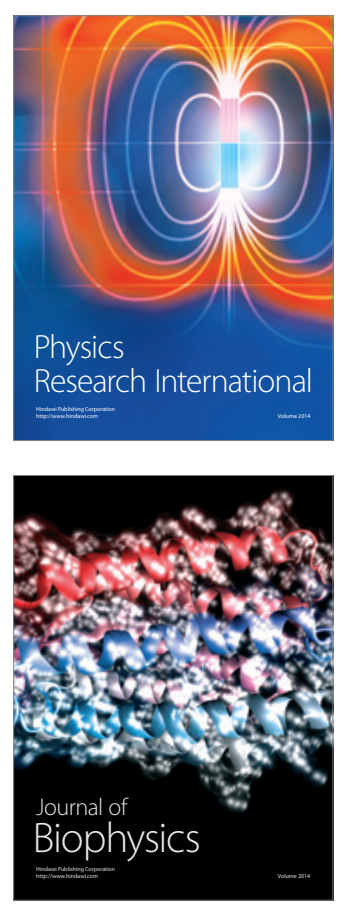
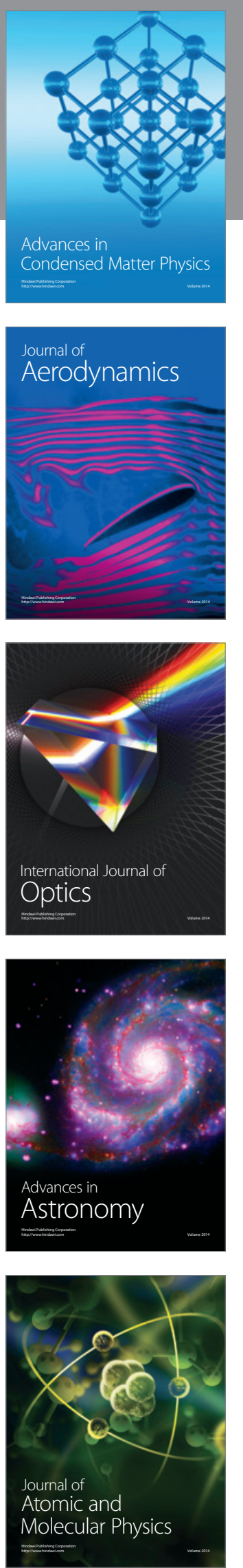